\title{
Theta Cone Metric Spaces and Some Fixed Point Theorems
}

\author{
Sahar Mohamed Ali Abou Bakr (D) \\ Department of Mathematics Faculty of Science, Ain Shams University Cairo, Cairo, Egypt \\ Correspondence should be addressed to Sahar Mohamed Ali Abou Bakr; saharm_ali@yahoo.com \\ Received 4 September 2020; Revised 21 October 2020; Accepted 23 October 2020; Published 16 November 2020 \\ Academic Editor: Jen-Chih Yao \\ Copyright (C) 2020 Sahar Mohamed Ali Abou Bakr. This is an open access article distributed under the Creative Commons \\ Attribution License, which permits unrestricted use, distribution, and reproduction in any medium, provided the original work is \\ properly cited. \\ This paper introduces the concept of the theta cone metric, studies its various topological properties, and gives some examples of \\ it. Furthermore, it proves some lemmas and then uses them to give further generalizations of some well-known fixed point \\ theorems. Specifically, Theorem 2 of the paper is a generalization of Reich's fixed point theorem.
}

\section{Introduction and Preliminaries}

In 2007, Huang and Zhang [1] introduced cone metric spaces as a generalization of metric spaces. Let $\mathscr{E}$ be a real Banach space, $C \subset \mathscr{E} . C$ is called a cone in $\mathscr{E}$ if

(1) $C$ is nonempty, closed, and $C \neq\{\Theta\}$, where $\Theta$ is zero (neutral element) of $\mathscr{E}$

(2) $\alpha C+\beta C \subset C$ for all nonnegative real numbers $\alpha, \beta$

(3) $C \cap-C=\{\Theta\}$

If int $C$ is the set of all interior points of $C$, then a cone $C$ in a normed space $\mathscr{E}$ induces the following ordered relations $[1,2]$ :

$$
\begin{aligned}
& u \prec v \Longleftrightarrow v-u \in C, \\
& u<v \Longleftrightarrow(v-u \in C \text { and } u \neq v), \\
& u_{\neq} \prec v \Longleftrightarrow v-u \in \operatorname{int} C .
\end{aligned}
$$

A sequence $\left\{w_{n}\right\}_{n \in N}$ in $\mathscr{E}$ is bounded above by $w \in \mathscr{E}$ iff

$$
w_{n} \prec_{\neq} w \quad \forall n \in \mathbb{N} \text {. }
$$

The cone $C$ is called normal if there is a number $M>0$ such that

$$
\forall u, v \in \mathscr{E}, \Theta \prec u \prec v \Longrightarrow\|u\| \leq M\|v\| .
$$

The least positive number satisfying the above is called the normal constant of $C$.
Huang and Zhang [1] supposed that $\mathscr{E}$ is a Banach space, $C$ is a cone in $\mathscr{E}$ with nonempty interior, and $\prec$ is partial ordering with respect to $C$. If $X$ is a nonempty set, the distance $d(x, y)$ of two elements $x$ and $y$ in the space $X$ is defined to be a vector in the cone $C$ of the ordered Banach space $(\mathscr{E}, \prec)$.

(1) $\Theta \prec \neq d(x, y) \forall x, y \in X$.

(2) $d(x, y)=\Theta \prec x=y$.

(3) $d(x, y)=d(y, x) \forall x, y \in X$.

(4) $d(x, z) \prec[d(x, y)+d(y, z)] \forall x, y, z \in X$.

The triple $(X, C, d)$ is known as the cone metric space. They carefully studied convergence and completeness and then proved some fixed point theorems for the contractive type of mappings in this setting.

In 2010, Haghi et al. [3] showed that some generalizations in fixed point theory are really consequences of Huang and Zhang results.

In contrast, in 2012, Cakally et al. [4] obtained that any cone metric space $(X, d)$ is equivalent to the usual metric space $\left(X, d^{\star}\right)$, where the real-valued metric function $d^{\star}$ is defined by a nonlinear scalarization function $[5,6]$.

In 2013, Liu and Xu [2] introduced the concept of cone metric spaces with Banach algebras; they replaced Banach spaces $\mathscr{E}$ by Banach algebras $\mathscr{A}$, and they proved some fixed point theorems of generalized Lipschitz mappings with weaker conditions on generalized Lipschitz constants (the 
constant $k$ is a vector in a normal cone of the Banach algebra, and the essential conditions on the contraction constant $k$ are neither order relations nor norm relations but spectrum radius). A well-formulated example shows that their main results concerning the fixed point theorems in the setting of cone metric spaces with Banach algebras are more useful than the standard results in cone metric spaces presented in the literature.

In 2013, Khojasteh et al. [7] proposed the notion of the $\theta$-metric as a proper generalization of a metric.

Definition 1. Let $\theta:[0, \infty) \times[0, \infty) \longrightarrow[0, \infty)$ be a continuous mapping with respect to both variables. Let $\operatorname{Im}(\theta)=\{\theta(s, t): s \geq 0, t \geq 0\}$. The mapping $\theta$ is called a $B$-action if and only if it satisfies the following conditions:

(1) $\theta(0,0)=0$ and $\theta(u, v)=\theta(v, u)$ for every $u, v \geq 0$.

(2)

$$
\theta(u, v)<\theta(w, t) \text { if either }\left\{\begin{array}{l}
u<w \text { and } v \leq t \\
\text { or } \\
u \leq w \text { and } v<t
\end{array}\right.
$$

(3) For every $u \in \operatorname{Im}(\theta)$ and every $v \in[0, u]$, there is $w \in[0, u]$ such that $\theta(v, w)=u$.

(4) $\theta(u, 0) \leq u$ for every $u>0$.

They functionally formulated the notion of $\theta$-metric spaces, and then, they gave the terminology of open and closed sets. Furthermore, they gave a detailed and comprehensive study of convergence and Cauchyness of sequences in this frame of work.

Now, we replace $[0, \infty)$ by a cone in a normed space and introduce the following analogous generalization of the definition of the $\theta$ function.

Definition 2. Let $(\mathscr{E}, \prec)$ be an ordered normed space, where $\prec$ is the ordered relation induced by a cone $C \subset \mathscr{E}$. Let $\theta: C \times C \longrightarrow C$ be a continuous mapping with respect to each variable. Let $\operatorname{Im}(\theta)=\left\{t: t \in C\right.$ such that $\exists u_{0}, v_{0} \in$ $\left.\mathscr{E}, \theta\left(u_{0}, v_{0}\right)=t\right\}$. A mapping $\theta$ is called an ordered action on $\mathscr{E}$ if and only if it satisfies the following conditions:

(1) $\theta(\Theta, \Theta)=\Theta$ and $\theta(u, v)=\theta(v, u)$ for every $u, v \in C$.

$$
\theta(u, v)<\theta(w, t) \text { if either }\left\{\begin{array}{l}
u<w, v \prec t \\
\text { or } \\
u \prec w, v<t
\end{array}\right.
$$

(3) For every $u \in \operatorname{Im}(\theta)$ and every $\Theta \prec v \prec u$, there is $\Theta \prec w \prec u$ such that $\theta(v, w)=u$.

(4) $\theta(u, \Theta) \prec u$ for every $u \in C /\{\Theta\}$.

Because $x-\Theta \in C$ for every $x \in C$, one can write instead $\Theta \prec x$ for every $x \in C,[\Theta<x$ for every $x \in C /\{\Theta\}]$.
Example 1. Let $\mathbb{R}^{m}$ be the vector space of all finite $m$ sequences of real numbers with the usual operations of addition and scalar multiplication, for $a=\left\{a_{k}\right\}_{k=1}^{m} \in \mathbb{R}^{m}$. Denote $\|a\|_{p}=\sqrt[p]{\sum_{k=1}^{m}\left|a_{k}\right|^{p}}$ and $\|a\|_{\infty}=\max _{k=1}^{m}\left|a_{k}\right|$. If $1 \leq p$, then $\left(\mathbb{R}^{m},\|.\|_{p}\right)$ is the Banach space. If $0 \leq p<1$, then $\left(\mathbb{R}^{m},\|\cdot\|_{p}\right)$ is the quasi-normed space.

Let $\mathscr{E}=\mathbf{M}_{\mathbf{n}}\left(\mathbb{R}^{m}\right)$ be the vector space of all $n$-square matrices whose entries are elements in $\mathbb{R}^{m}$ :

$$
\mathscr{E}=\left\{\begin{array}{c}
u: u=\left\{u_{i j}\right\}_{1 \leq i, j \leq n} \\
u_{i j}=\left\{u_{k}^{i j}\right\}_{k=1}^{m} \in \mathbb{R}^{m}, \quad \forall 1 \leq i, j \leq n
\end{array}\right\} .
$$

Operations on $\mathscr{E}$ are defined as follows:

$$
\begin{array}{r}
\alpha u=\alpha\left\{u_{i j}\right\}_{1 \leq i, j \leq n}=\left\{\left\{\alpha u_{k}^{i j}\right\}_{k=1}^{m}\right\}_{1 \leq i, j \leq n}, \\
u \oplus v=\left\{u_{i j}\right\}_{1 \leq i, j \leq n} \oplus\left\{v_{i j}\right\}_{1 \leq i, j \leq n}=\left\{\left\{u_{k}^{i j}+v_{k}^{i j}\right\}_{k=1}^{m}\right\}_{1 \leq i, j \leq n} .
\end{array}
$$

The space $\mathscr{E}$ is a Banach space endowed with the following norms:

$$
\begin{array}{r}
\|u\|_{p}=\sum_{1 \leq i, j \leq n}\left\|\left\{u_{k}^{i j}\right\}_{k=1}^{m}\right\|_{p}, \\
\|u\|_{\infty}=\max _{1 \leq i \leq n} \sum_{1 \leq j \leq n}\left\|\left\{u_{k}^{i j}\right\}_{k=1}^{m}\right\|_{p} .
\end{array}
$$

The zero element of the space $\mathscr{E}$, is the matrix $\theta$, and every entry of the matrix $\theta$ is the all of its entries are the zero element of $\mathbb{R}^{m}, 0=\{0,0, \ldots, 0\}$.

Let $C=\left\{u: u=\left\{\left\{u_{k}^{i j}\right\}_{k=1}^{m}\right\}_{1 \leq i, j \leq n}, 1 \leq i, j \leq n\right.$, and $u_{k}^{i j} \geq$ 0 for all $1 \leq i, j \leq n$, and $1 \leq k \leq m\}$, Each entry is a vector of the space $\mathbb{R}^{m}$, and the entries of these vectors are nonnegative real numbers. Then, $C$ is a cone in $\mathscr{E}$. The cone $C$ induces the ordered relation $u=\left\{\left\{u_{k}^{i j}\right\}_{k=1}^{m}\right\}_{1 \leq i, j \leq n} \prec v=$ $\left\{\left\{v_{k}^{i j}\right\}_{k=1}^{m}\right\}_{1 \leq i, j \leq n}$ if and only if $v_{k}^{i j} \geq u_{k}^{i j}$ for every $k \in$ $\{1,2, \ldots, m\}$ and every $i, j, 1 \leq i, j \leq n$.

Now, let $k$ be nonnegative real number such that $0 \leq k<1$. Define $\theta_{q}: C \times C \longrightarrow C, q \in\{1,2,3,4,5\}$ by

$$
\begin{aligned}
& \theta_{1}(u, v)=[u \oplus v]=\left\{\left\{u_{k}^{i j}+v_{k}^{i j}\right\}_{k=1}^{m}\right\}_{1 \leq i, j \leq n}, \\
& \theta_{2}(u, v)=k[u \oplus v]=\left\{\left\{k\left(u_{k}^{i j}+v_{k}^{i j}\right)\right\}_{k=1}^{m}\right\}_{1 \leq i, j \leq n}, \\
& \theta_{3}(u, v)=\left\{\left\{k\left(u_{k}^{i j}+v_{k}^{i j}+u_{k}^{i j} \times v_{k}^{i j}\right)\right\}_{k=1}^{m}\right\}_{1 \leq i, j \leq n}, \\
& \theta_{4}(u, v)=\left\{\left\{u_{k}^{i j}+v_{k}^{i j}+u_{k}^{i j} \times v_{k}^{i j}\right\}_{k=1}^{m}\right\}_{1 \leq i, j \leq n}, \\
& \theta_{5}(u, v)=\left\{\left\{\left(u_{k}^{i j}+v_{k}^{i j}\right)\left(1+u_{k}^{i j} \times v_{k}^{i j}\right)\right\}_{k=1}^{m}\right\}_{1 \leq i, j \leq n} .
\end{aligned}
$$

Then, the functions $\theta_{q}, q=1,2, \ldots, 5$, are all ordered actions on $\mathscr{E}$.

Furthermore, we replace $[0, \infty)$ by a cone in a normed space and use ordered actions to introduce the concept of action function cone metric spaces.

Definition 3. Let $(\mathscr{E}, \prec)$ be an ordered normed space, where $\prec$ is the ordered relation induced by a cone $C \subset \mathscr{E}, X$ be a 
nonempty set, and $\theta$ be an ordered action on $\mathscr{E}$. Then, the function $d_{\theta}: X \times X \longrightarrow C$ is called $\theta$-cone-metric on $X$ if and only if $d_{\theta}$ satisfies the following conditions:

(1) $d_{\theta}(x, y)=\Theta \Longleftrightarrow x=y$.

(2) $d_{\theta}(x, y)=d_{\theta}(y, x) \forall x, y \in X$.

(3) $d_{\theta}(x, y) \prec \theta\left(d_{\theta}(x, z), d_{\theta}(z, y)\right) \forall x, y, z \in X$.

The triple $\left(X, C, d_{\theta}\right)$ is defined to be a $\theta$-cone-metric space or equivalently action function cone metric space.
Remark 1. We mention that the class of metric spaces is included in the class of $\theta$-metric spaces if we consider $\theta(u, v)=u+v, u, v \in[0, \infty)$. Also, we mention that the class of $\theta$-metric spaces is included in the class of $\theta$-cone-metric spaces if we take $(\mathscr{E}, \prec)=([0, \infty), \leq)$.

Example 2. Let $\mathscr{E}, C$, and $\theta_{1}$ be given as in Example $1 X=$ $\mathbf{M}_{\mathbf{n}}\left(\mathbb{R}^{m}\right)$ be the space of all $n \times n$ matrices whose entries are elements of the space $\mathbb{R}^{m}$. Then, $X$ is a nonempty set. The function $d_{\theta_{1}}, d_{\theta_{1}}: X \times X \longrightarrow C$, defined by

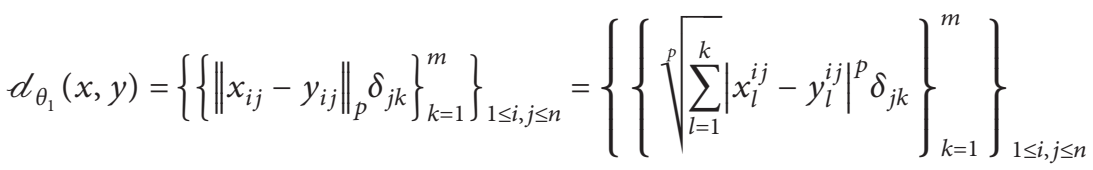

is a $\theta_{1}$-cone metric on $X$, and $\left(X, C, d_{\theta_{1}}\right)$ is the $\theta_{1}$-conemetric space. In fact, conditions (1) and (2) are clear, and for any $x, y, z \in X$, we have

$$
\begin{aligned}
& {\left[d_{\theta_{1}}(x, z)+d_{\theta_{1}}(z, y)\right]=\left[\left\{\left\{\left\|x_{i j}-z_{i j}\right\|_{p} \delta_{j k}\right\}_{k=1}^{m}\right\}_{1 \leq i, j \leq n}+\left\{\left\{\left\|z_{i j}-y_{i j}\right\|_{p} \delta_{j k}\right\}_{k=1}^{m}\right\}_{1 \leq i, j \leq n}\right]} \\
& =\left[\left\{\left\{\left\|x_{i j}-z_{i j}\right\|_{p} \delta_{j k}\right\}_{k=1}^{m}+\left\{\left\|z_{i j}-y_{i j}\right\|_{p} \delta_{j k}\right\}_{k=1}^{m}\right\}_{1 \leq i, j \leq n}\right]=\left\{\left\{\left[\left\|x_{i j}-z_{i j}\right\|_{p}+\left\|z_{i j}-y_{i j}\right\|_{p}\right] \delta_{j k}\right\}_{k=1}^{m}\right\}_{1 \leq i, j \leq n}
\end{aligned}
$$

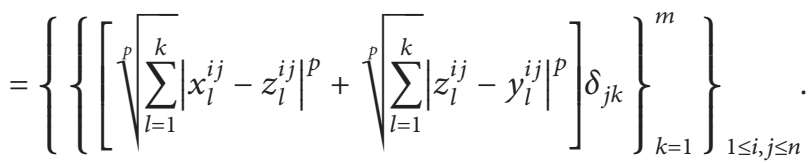

Hence, $d_{\theta_{1}}(x, y) \prec \theta_{1}\left(d_{\theta_{1}}(x, z), d_{\theta_{1}}(z, y)\right) \forall x, y, x \in X$ because

$$
\begin{aligned}
& \sqrt[p]{\sum_{l=1}^{k}}\left|x_{l}^{i j}-y_{l}^{i j}\right|^{p} \leq\left[\sqrt[p]{\sum_{l=1}^{k}}\left|x_{l}^{i j}-z_{l}^{i j}\right|^{p}+\sqrt[p]{\sum_{l=1}^{k}}\left|z_{l}^{i j}-y_{l}^{i j}\right|^{p}\right], \\
& \forall 1 \leq i, j \leq n, 1 \leq k \leq m \text {. }
\end{aligned}
$$

Example 3. Let $\mathscr{E}, C$, and $\theta_{4}$ be given as in Example 1 and $X=\{x, y, z\}$ be three elements. Then, the function $d_{\theta_{4}}$, $d_{\theta_{4}}: X \times X \longrightarrow C$, is defined by

$$
\begin{aligned}
& d_{\theta_{4}}(x, x)=d_{\theta_{4}}(y, y)=d_{\theta_{4}}(z, z)=\Theta, \\
& d_{\theta_{4}}(x, y)=d_{\theta_{4}}(y, x)=\left\{u_{i j}\right\}_{1 \leq i, j \leq n}, \quad \text { where } u_{11}=\{2,0,0, \ldots\}, u_{i j}=\{0,0,0, \ldots, 0\} \forall 1<i, j \leq n, \\
& d_{\theta_{4}}(y, z)=d_{\theta_{4}}(z, y)=\left\{v_{i j}\right\}_{1 \leq i, j \leq n}, \quad \text { where } v_{11}=\{3,0,0, \ldots\}, v_{i j}=\{0,0,0, \ldots, 0\} \forall 1<i, j \leq n, \\
& d_{\theta_{4}}(x, z)=d_{\theta_{4}}(z, x)=\left\{w_{i j}\right\}_{1 \leq i, j \leq n}, \quad \text { where } w_{11}=\{6,0,0, \ldots\}, w_{i j}=\{0,0,0, \ldots, 0\} \forall 1<i, j \leq n .
\end{aligned}
$$

Note that $d_{\theta_{4}}$ is not a metric on $X$ because $d_{\theta_{4}}(x, z)>d_{\theta_{4}}(x, y)+d_{\theta_{4}}(y, z)$, but it is a $\theta_{4}$-cone metric on $X$, and $\left(X, C, d_{\theta_{4}}\right)$ is a $\theta_{4}$-cone-metric space.
In 2017, J. Fernandez et al. introduced F-cone-metric spaces over Banach algebra and gave some generalization of some previous fixed point theorems. 
In 2017, Suzuki [8] introduced different generalized approaches for the strongest sequentially compatible topology on a $\nu$-generalized metric space and studied its characterizations.

In 2019, Abou Bakr [9] gave some common fixed point theorems (and, in particular, fixed points) of the generalized contraction type of cyclic mappings defined on cone metric spaces on Banach algebra.

In 2020, Abou Bakr [10] gave a study on the common fixed point of joint generalized types of contraction mappings in quasi-metric spaces.

\section{Main Results}

We have the following two sections.

2.1. Convergence and Cauchyness in $\theta$-Cone-Metric Spaces. Let us start with the following definition.

Definition 4. Let $\left(X, C, d_{\theta}\right)$ be a $\theta$-cone-metric space. An open ball $N_{d_{\theta}}(x, u)$ at center $x \in X$ with a radius $u \in \operatorname{Im}(\theta)$ (neighborhood of $x$ at radius $u$ ) is defined as

$$
N_{d_{\theta}}(x, u)=:\left\{y: y \in X, d_{\theta}(x, y)<u\right\} .
$$

A subset $Y \subset X$ is bounded if and only if there are $x \in X$ and $u \in \operatorname{Im}(\theta)$ such that $Y \subset N_{d_{\theta}}(x, u)$. Limit and interior points are defined in the usual way. Additionally, bounded, open, and closed sets in $X$ are also defined.

Remark 2. A sequence $\left\{x_{n}\right\}_{n \in \mathbb{N}}$ in $\left(X, C, d_{\theta}\right)$ converges to $x$ whenever for each $u \in \operatorname{Im}(\theta)$ with $\Theta<u$, there is $n_{0} \in \mathbb{N}$ such that $d_{\theta}\left(x_{n}, x\right)<u$ for all $n \geq n_{0}$. We instead write $x_{n} \longrightarrow{ }_{n \rightarrow \infty} x$.

A sequence $\left\{x_{n}\right\}_{n \in \mathbb{N}}$ in $\left(X, C, d_{\theta}\right)$ is Cauchy whenever for each $u \in C$ with $\Theta<u$, there is $n_{0} \in \mathbb{N}$ such that $d_{\theta}\left(x_{n}, x_{m}\right)<u$ for all $n, m \geq n_{0}$.

A sequence $\left\{x_{n}\right\}_{n \in \mathbb{N}}$ in $\left(X, C, d_{\theta}\right)$ is bounded if and only if there are $x \in X$ and $u \in \operatorname{Im}(\theta)$ such that $\left\{x_{n}\right\}_{n \in \mathbb{N}} \subset N_{d_{\theta}}(x, u)$. Equivalently, a sequence $\left\{x_{n}\right\}_{n \in \mathbb{N}}$ in $\left(X, C, d_{\theta}\right)$ is bounded if and only if there are $x \in X$ and $u \in \operatorname{Im}(\theta)$ such that $d_{\theta}\left(x_{n}, x\right)<u$ for all $n \in \mathbb{N}$.

We have the following lemmas.

Lemma 1. Every neighborhood in $\left(X, C, d_{\theta}\right)$ is an open subset.

Proof. Let $x \in X$ be an arbitrary element and $N_{d_{\theta}}(x, u)$ be any neighborhood of $x$ with any radius $u \in C$; we show that, for every $y \in N_{d_{\theta}}(x, u)$, there is some $w \in \operatorname{Im}(\theta)$ such that $N_{d_{\theta}}(y, w) \subset N_{d_{\theta}}(x, u)$. Set $v=d_{\theta}(x, y)$, and we have $u \in \operatorname{Im}(\theta)$ and $\Theta \prec v \prec u$. Using the definition of $\theta$, there is $w$, $\Theta \prec w \prec u$, such that $\theta(v, w)=u$. We claim that $N_{d_{\theta}}(y, w)$ is the required neighborhood. In fact, if $z \in N_{d_{\theta}}(y, w)$ is an arbitrary element, then $d_{\theta}(y, z)<w$. Now, using the definition of $d_{\theta}$ and then the definition of $\theta$, we have the following:

$$
d_{\theta}(x, z) \prec \theta\left(d_{\theta}(x, y), d_{\theta}(y, z)\right)<\theta(v, w)=u .
$$

Hence, $z \in N_{d_{\theta}}(x, u)$; this completes the proof.

Lemma 2. A sequence $\left\{x_{n}\right\}_{n \in \mathbb{N}}$ in $\left(X, C, d_{\theta}\right)$ converges to $x$ if and only if $d_{\theta}\left(x_{n}, x\right) \longrightarrow_{n \longrightarrow \infty} \Theta$ converges in $\mathscr{E}$.

Proof. Suppose that $x_{n} \longrightarrow{ }_{n}^{d_{\theta}} \longrightarrow \infty$, and let $\varepsilon>0$. Then, choose $u \in \operatorname{Im}(\theta), \Theta<u$ with $M\|u\|<\varepsilon$, where $M$ is the normal constant of $C$; for this $u$, there is $n_{0} \in \mathbb{N}$ such that $d_{\theta}\left(x_{n}, x\right)<u$ for all $n \geq n_{0}$; hence, $\left\|d_{\theta}\left(x_{n}, x\right)\right\|<M\|u\|$ for all $n \geq n_{0}$, and accordingly, we have $\left\|d_{\theta}\left(x_{n}, x\right)\right\|<\varepsilon$ for all $n \geq n_{0}$. This proves that $d_{\theta}\left(x_{n}, x\right) \longrightarrow_{n \rightarrow \infty} \Theta$.

Conversely, let $d_{\theta}\left(x_{n}, x\right) \longrightarrow n \rightarrow \infty \infty\left[\left\|d_{\theta}\left(x_{n}, x\right)\right\|\right.$ $\longrightarrow{ }_{n \rightarrow \infty} 0$ ]; we show that $x_{n} \longrightarrow{ }_{n \rightarrow \infty}{ }_{n} x$. Let $u \in \operatorname{Im}(\theta)$ be arbitrary; since $\theta$ is continuous, there is a neighborhood of $u$ with some radius $\varepsilon>0, N_{\varepsilon}(u)$ in $\mathscr{E}$ and $N_{\varepsilon}(u) \subset C$; for this $\varepsilon$, there is a natural number $n_{0} \in \mathcal{N}$ such that $\left\|d_{\theta}\left(x_{n}, x\right)\right\|<\varepsilon$ for every $n \geq n_{0}$, and since $d_{\theta}\left(x_{n}, x\right)=u-\left[u-d_{\theta}\left(x_{n}, x\right)\right]$, we see that $\left\|u-\left[u-d_{\theta}\left(x_{n}, x\right)\right]\right\|<\varepsilon$ for every $n \geq n_{0}$; hence, $\left[u-d_{\theta}\left(x_{n}, x\right)\right] \in N_{\varepsilon}(u)$, and consequently, $u-d_{\theta}\left(x_{n}, x\right)$ $\in C$, that is, $d_{\theta}\left(x_{n}, x\right) \prec u$. This proves that $x_{n} \longrightarrow \stackrel{d}{d_{\theta}} \rightarrow \infty x$.

Lemma 3. The limit of any sequence $\left\{x_{n}\right\}_{n \in \mathbb{N}}$ in $\left(X, C, d_{\theta}\right)$ is unique.

Proof. Suppose that $x_{n} \longrightarrow{ }_{n}^{d_{\theta}} \longrightarrow \infty x$ and $x_{n} \longrightarrow_{n \rightarrow \infty}^{d_{\theta}} y$; we show that $x=y$. Using Lemma 2, we have $d_{\theta}\left(x_{n}, x\right) \longrightarrow_{n \rightarrow \infty} \Theta$ and $d_{\theta}\left(x_{n}, y\right) \longrightarrow_{n \rightarrow \infty} \Theta$, and using the definition of $d_{\theta}$ and the continuity of $\theta$, we have

$$
\Theta \prec d_{\theta}(x, y) \prec \theta\left(d_{\theta}\left(x_{n}, x\right), d_{\theta}\left(x_{n}, y\right)\right) \underset{n \longrightarrow \infty}{\longrightarrow} \theta(\Theta, \Theta)=\Theta .
$$

Hence, $d_{\theta}(x, y)=\Theta$, that is, $x=y$.

Lemma 4. Let $\left(X, C, d_{\theta}\right)$ be a $\theta$-cone-metric space, $x_{n} \longrightarrow{ }_{n} \longrightarrow \infty x$, and $y_{n} \longrightarrow{ }_{n} \longrightarrow \infty y$. Then,

$$
d_{\theta}\left(x_{n}, y_{n}\right) \underset{n \longrightarrow \infty}{\longrightarrow} d_{\theta}(x, y)
$$

Proof. Using Lemma 4, we have $d_{\theta}\left(x_{n}, x\right) \longrightarrow{ }_{n \rightarrow \infty} \Theta$ and $d_{\theta}\left(y_{n}, y\right) \longrightarrow_{n \rightarrow \infty} \Theta \quad$ since $\quad d_{\theta}\left(x, y_{n}\right) \prec \theta\left(d_{\theta}(x, y)\right.$, $\left.d_{\theta}\left(y, y_{n}\right)\right)$ and $\theta$ is continuous; then, conditions (2) and (4) of $\theta$ imply the following:

$$
\begin{aligned}
& d_{\theta}\left(x, y_{n}\right) \prec \theta\left(d_{\theta}(x, y), d_{\theta}\left(y, y_{n}\right)\right) \\
& \longrightarrow \longrightarrow \theta\left(d_{\theta}(x, y), \Theta\right) \prec d_{\theta}(x, y),
\end{aligned}
$$

and consequently,

$$
\begin{aligned}
d_{\theta}\left(x_{n}, y_{n}\right) & \leqslant \theta\left(d_{\theta}\left(y_{n}, x\right), d_{\theta}\left(x, x_{n}\right)\right) \text { use the continuity of } \theta \\
& \longrightarrow \theta\left(d_{\theta}(x, y), \Theta\right) \text { use condition }(4) \text { of } \theta \\
& \preccurlyeq d_{\theta}(x, y),
\end{aligned}
$$

Using the continuity of $\theta$ once more gives 


$$
\begin{aligned}
d_{\theta}(x, y) & \prec \theta\left(d_{\theta}\left(x, x_{n}\right), \theta\left(d_{\theta}\left(x_{n}, y_{n}\right), d_{\theta}\left(y_{n}, y\right)\right)\right) \\
& \longrightarrow \lim _{n} \theta\left(d_{\theta}\left(x, x_{n}\right), \theta\left(d_{\theta}\left(x_{n}, y_{n}\right), d_{\theta}\left(y_{n}, y\right)\right)\right) \\
& \prec \theta\left(\lim _{n \longrightarrow \infty} d_{\theta}\left(x, x_{n}\right), \lim _{n \longrightarrow \infty} \theta\left(d_{\theta}\left(x_{n}, y_{n}\right), d_{\theta}\left(y_{n}, y\right)\right)\right) \\
& \prec \theta\left(\lim _{n \longrightarrow \infty} d_{\theta}\left(x, x_{n}\right), \theta\left(\lim _{n \longrightarrow \infty} d_{\theta}\left(x_{n}, y_{n}\right), \lim _{n \longrightarrow \infty} d_{\theta}\left(y_{n}, y\right)\right)\right) \\
& \prec \theta\left(\Theta, \theta\left(\lim _{n \longrightarrow \infty} d_{\theta}\left(x_{n}, y_{n}\right), \Theta\right)\right)<\theta\left(\lim _{n \longrightarrow \infty} d_{\theta}\left(x_{n}, y_{n}\right), \Theta\right) \\
& \prec \lim _{n \longrightarrow \infty} d_{\theta}\left(x_{n}, y_{n}\right) .
\end{aligned}
$$

Using inequalities (19) and (20) gives $d_{\theta}\left(x_{n}, y_{n}\right) \longrightarrow$ $n \rightarrow \infty d_{\theta}(x, y)$ and completes the proof.

Lemma 5. A sequence $\left\{x_{n}\right\}_{n \in \mathbb{N}}$ in $\left(X, C, d_{\theta}\right)$ is Cauchy if and only if

$$
d_{\theta}\left(x_{n}, x_{m}\right) \underset{n, m \longrightarrow \infty}{\longrightarrow} \Theta .
$$

Proof. Suppose that $\left\{x_{n}\right\}_{n \in \mathbb{N}}$ is Cauchy, and let $\varepsilon>0$. Then, choose $u \in \operatorname{Im}(\theta), \Theta<u$ with $M\|u\|<\varepsilon$, where $M$ is the normal constant of $C$; for this $u$, there is $n_{0} \in \mathbb{N}$ such that $d_{\theta}\left(x_{n}, x_{m}\right)<u$ for all $n, m \geq n_{0}$; hence, $\left\|d_{\theta}\left(x_{n}, x_{m}\right)\right\|<M\|u\|$ for all $n, m \geq n_{0}$, and accordingly, we have $\left\|d_{\theta}\left(x_{n}, x_{m}\right)\right\|<\varepsilon$ for all $n, m \geq n_{0}$. This proves that $d_{\theta}\left(x_{n}, x_{m}\right) \longrightarrow_{n, m \rightarrow \infty} \Theta$.

Conversely, let $d_{\theta}\left(x_{n}, x_{m}\right) \longrightarrow_{n, m \rightarrow \infty} \Theta\left[\left\|d_{\theta}\left(x_{n}, x_{m}\right)\right\|\right.$ $\left.\longrightarrow{ }_{n, m \longrightarrow \infty} 0\right]$; we show that $\left\{x_{n}\right\}_{n \in \mathbb{N}}$ is Cauchy. Let $u \in \operatorname{Im}(\theta)$ be arbitrary; since $\theta$ is continuous, there is a neighborhood of $u$ with some radius $\varepsilon>0, N_{\varepsilon}(u)$ in $\mathscr{E}$ such that $N_{\varepsilon}(u) \subset C$; for this $\varepsilon$, there is a natural number $n_{0} \in \mathcal{N}$ such that $\left\|d_{\theta}\left(x_{n}, x_{m}\right)\right\|<\varepsilon$ for every $n, m \geq n_{0}$, and since $d_{\theta}\left(x_{n}, x_{m}\right)=u-\left[u-d_{\theta}\left(x_{n}, x_{m}\right)\right]$, we see that $\left\|u-\left[u-d_{\theta}\left(x_{n}, x_{m}\right)\right]\right\|<\varepsilon$ for every $n, m \geq n_{0}$. Hence, $\left[u-d_{\theta}\left(x_{n}, x_{m}\right)\right] \in N_{\varepsilon}(u)$, and consequently, $u-d_{\theta}($ $\left.x_{n}, x_{m}\right) \in C$, that is, $d_{\theta}\left(x_{n}, x_{m}\right) \prec u$ for every $n, m \geq n_{0}$. This proves that $\left\{x_{n}\right\}_{n \in \mathbb{N}}$ is Cauchy.

Lemma 6. Every convergent sequence in the $\theta$-cone-metric space is Cauchy.

Proof. Let $\left\{x_{n}\right\}_{n \in \mathbb{N}}$ in $\left(X, C, d_{\theta}\right)$ converge to $x$, and let $m, n \in \mathbb{N}$ with $m \geq n$. Then, the continuity of $\theta$ insures the following:

$$
d_{\theta}\left(x_{n}, x_{m}\right) \prec \theta\left(d_{\theta}\left(x, x_{n}\right), d_{\theta}\left(x, x_{m}\right)\right) \underset{n, m \longrightarrow \infty}{\longrightarrow} \theta(\Theta, \Theta)=\Theta .
$$

The following lemmas are mainly used in the upcoming generalizations of fixed point theorems.

Lemma 7. Let $\left(X, C, d_{\theta}\right)$ be a $\theta$-cone-metric space and $\left\{x_{n}\right\}_{n \in N}$ be in $X$ such that $\left\{d_{\theta}\left(x_{n}, x_{n+1}\right)\right\}_{n \in \mathbb{N}}$ converges to $\Theta$. Then, $\left\{x_{n}\right\}_{n \in \mathbb{N}}$ is a Cauchy sequence.

Proof. Suppose that $\left\{x_{n}\right\}_{n \in \mathbb{N}}$ is not Cauchy; then, there exist $u \in \operatorname{Im}(\theta), \Theta<u$, and sequences $\{J(n)\}_{n \in \mathbb{N}}$ and $\{k(n)\}_{n \in \mathbb{N}}$ of natural numbers such that, for any $J(n)>k(n)>n$,

$$
u \prec d_{\theta}\left(x_{J(n)}, x_{k(n)}\right) \text { and } d_{\theta}\left(x_{J(n)-1}, x_{k(n)}\right)<u \quad \forall n \in \mathbb{N} \text {. }
$$

Using the definition and the continuity of $\theta$ and the fact that any subsequence of $\left\{d_{\theta}\left(x_{n}, x_{n+1}\right)\right\}_{n \in \mathbb{N}}$ converges to $\Theta$, we have the following contradiction:

$$
\begin{aligned}
u & <d_{\theta}\left(x_{J(n)}, x_{k(n)}\right) \\
& <\theta\left(d_{\theta}\left(x_{J(n)-1}, x_{k(n)}\right), d_{\theta}\left(x_{J(n)}, x_{j(n)-1}\right)\right) \\
& <\theta\left(u, d_{\theta}\left(x_{J(n)}, x_{j(n)-1}\right)\right) \\
& <\theta\left(u, \lim _{n \longrightarrow \infty} d_{\theta}\left(x_{J(n)}, x_{j(n)-1}\right)\right) \\
& =\theta(u, \Theta)<u .
\end{aligned}
$$

Lemma 8. Let $\left(X, C, d_{\theta}\right)$ be a $\theta$-cone-metric space and $\left\{x_{n}\right\}_{n \in N}$ be in $X$ such that

$$
d_{\theta}\left(x_{n+2}, x_{n+1}\right) \prec t_{0} d_{\theta}\left(x_{n+1}, x_{n}\right), \quad n=0,1,2, \ldots,
$$

for some real number $t_{0}, 0 \leq t_{0}<1$. Then, $\left\{x_{n}\right\}_{n \in N}$ is Cauchy.

Proof. Taking into account the conditions $\alpha C \subset C$ for every nonnegative real number $\alpha, C+C \subset C$, and $d_{\theta}\left(x_{n+1}, x_{n}\right) \prec$ $t_{0} d_{\theta}\left(x_{n}, x_{n-1}\right)$ imply

$$
t_{0} d_{\theta}\left(x_{n}, x_{n-1}\right)-d_{\theta}\left(x_{n+1}, x_{n}\right) \in C .
$$

Hence,

$$
t_{0}\left[t_{0} d_{\theta}\left(x_{n}, x_{n-1}\right)-d_{\theta}\left(x_{n+1}, x_{n}\right)\right] \in C,
$$

that is,

$$
t_{0}^{2} d_{\theta}\left(x_{n}, x_{n-1}\right)-t_{0} d_{\theta}\left(x_{n+1}, x_{n}\right) \in C .
$$

Additionally, $d_{\theta}\left(x_{n+2}, x_{n+1}\right) \prec t_{0} d_{\theta}\left(x_{n+1}, x_{n}\right)$ gives

$$
t_{0} d_{\theta}\left(x_{n+1}, x_{n}\right)-d_{\theta}\left(x_{n+2}, x_{n+1}\right) \in C \text {. }
$$

Inclusions (28) and (29) yield the following:

$$
\begin{array}{r}
{\left[t_{0}^{2} d_{\theta}\left(x_{n}, x_{n-1}\right)-t_{0} d_{\theta}\left(x_{n+1}, x_{n}\right)\right]+\left[t_{0} d_{\theta}\left(x_{n+1}, x_{n}\right)\right.} \\
\left.-d_{\theta}\left(x_{n+2}, x_{n+1}\right)\right] \in C .
\end{array}
$$

Thus,

$$
\left[t_{0}^{2} d_{\theta}\left(x_{n}, x_{n-1}\right)-d_{\theta}\left(x_{n+2}, x_{n+1}\right)\right] \in C,
$$

and hence,

$$
d_{\theta}\left(x_{n+2}, x_{n+1}\right) \prec t_{0} d_{\theta}\left(x_{n+1}, x_{n}\right) \prec t_{0}^{2} d_{\theta}\left(x_{n}, x_{n-1}\right) .
$$

This implies successively

$$
\begin{aligned}
d_{\theta}\left(x_{n}, x_{n+1}\right) & \prec t_{0} d_{\theta}\left(x_{n-1}, x_{n}\right) \\
& \prec t_{0}^{2} d_{\theta}\left(x_{n-2}, x_{n-1}\right) \\
& \cdots \\
& \prec t_{0}^{n} d_{\theta}\left(x_{0}, x_{1}\right) .
\end{aligned}
$$


Since $0 \leq t_{0}<1$, the sequence $\left\{t_{0}^{n}\right\}_{n \in N}$ is convergent to 0 , and taking the limit as $n \longrightarrow \infty$ of the two sides of (33) shows that $t_{0}^{n} d_{\theta}\left(x_{1}, x_{0}\right) \longrightarrow \Theta$ as $n \longrightarrow \infty$; hence, $\lim _{n \longrightarrow \infty} d_{\theta}\left(x_{n}, x_{n+1}\right)=\Theta$. Using Lemma 7 shows that $\left\{x_{n}\right\}_{n \in N}$ is Cauchy and completes the proof.

\subsection{Fixed Point Theorem for Contraction Types of Mappings.} We have the following definition.

Definition 5. A $\theta$-cone-metric space $\left(X, C, d_{\theta}\right)$ is complete whenever every Cauchy sequence converges to an element belonging to it.

Banach's contraction principle [11] is one of the pivotal results of nonlinear analysis and its applications, which establishes that every contraction mapping defined on a complete metric space has a unique fixed point. In this paper, we use the concept of $\theta$-cone-metric spaces to generalize the Banach contraction principle as follows.

Theorem 1. Let $\left(X, C, d_{\theta}\right)$ be a complete $\theta$-cone-metric space on a normed space $\mathscr{E}$ and $S: X \longrightarrow X$ be a mapping that satisfies the following:

$$
d_{\theta}(S(x), S(y)) \prec t_{0} d_{\theta}(x, y), \quad \forall x, y \in X,
$$

for some real number $t_{0}, 0 \leq t_{0}<1$. Then, $S$ has a unique fixed point.

Proof. Let $x_{0} \in X$ be a given element; then, the iterated sequence $\left\{S^{n}\left(x_{0}\right)\right\}_{n \in \mathbb{N}}, x_{n}=S^{n}(x)$, satisfies the following:

$$
\begin{aligned}
d_{\theta}\left(x_{n+2}, x_{n+1}\right) & =d_{\theta}\left(S\left(x_{n+1}\right), S\left(x_{n}\right)\right) \\
& \prec t_{0} d_{\theta}\left(x_{n+1}, x_{n}\right), \quad n=0,1,2, \ldots .
\end{aligned}
$$

Using Lemma 8, the sequence of iterates is a Cauchy sequence. According to the completeness of $X$, there exists $x \in X$ such that $x_{n} \longrightarrow{ }_{n}^{d_{\theta}} \longrightarrow \infty x$; we claim that $x$ is a fixed point of $S$. In fact, we have

$$
\begin{aligned}
d_{\theta}\left(x_{n+1}, S(x)\right) & =d_{\theta}\left(S\left(x_{n}\right), S(x)\right), \quad n=0,1,2, \ldots \\
& \prec t_{0} d_{\theta}\left(x_{n}, x\right) \underset{n \longrightarrow \infty}{\longrightarrow} \Theta .
\end{aligned}
$$

It means that $x_{n} \longrightarrow{ }_{n}^{d_{\theta}} \longrightarrow \infty S(x)$; using Lemma 3 proves that $S(x)=x$. Finally, we prove that $x$ is the unique fixed point of $S$. Suppose, on the contrary, that $x$ and $y$ are two distinct fixed points of $S$; then, $d_{\theta}(x, y) \neq \Theta$, and on the other side, $\left(1-t_{0}\right) d_{\theta}(x, y) \in C$ because $\left(1-t_{0}\right)>0$, that is, $t_{0} d_{\theta}(x, y)<d_{\theta}(x, y)$. So, we get the following contradiction:

$$
d_{\theta}(x, y)=d_{\theta}(S(x), S(y)) \prec t_{0} d_{\theta}(x, y)<d_{\theta}(x, y) .
$$

One of beautiful generalizations of the Banach contraction principle was found by Reich [12] and further generalized by Hardy and Rogers [13]. In this paper, in case of the above introduced $\theta$-cone-metric space setting, we give the following further generalization.
Theorem 2. Let $\left(X, C, d_{\theta}\right)$ be a complete $\theta$-cone-metric space on a normed space $\mathscr{E}$ and $S: X \longrightarrow X$ be a mapping that satisfies the following:

$$
\begin{aligned}
d_{\theta}(S(x), S(y)) \prec & a d_{\theta}(x, y)+b d_{\theta}(x, S(x)) \\
& +c d_{\theta}(y, S(y)), \quad \forall x, y \in X,
\end{aligned}
$$

for some real numbers $a, b, c, 0<a+b+c<1$. Then, $S$ has a unique fixed point.

Proof. Let $x_{0} \in X$ be a given element. Then, the iterated sequence $\left\{S^{n}\left(x_{0}\right)\right\}_{n \in \mathbb{N}}, x_{n}=S^{n}(x)$, satisfies the following:

$$
\begin{aligned}
d_{\theta}\left(x_{n+2}, x_{n+1}\right)= & d_{\theta}\left(S\left(x_{n+1}\right), S\left(x_{n}\right)\right), \quad n=0,1,2, \ldots \\
\prec & a d_{\theta}\left(x_{n+1}, x_{n}\right)+b d_{\theta}\left(x_{n+1}, S\left(x_{n+1}\right)\right) \\
& +c d_{\theta}\left(x_{n}, S\left(x_{n}\right)\right) \\
\prec & {[a+c] d_{\theta}\left(x_{n+1}, x_{n}\right)+b d_{\theta}\left(x_{n+1}, x_{n+2}\right), }
\end{aligned}
$$

and consequently, $[a+c] d_{\theta}\left(x_{n+1}, x_{n}\right)-[1-b] d_{\theta}\left(x_{n+2}\right.$, $\left.x_{n+1}\right) \in C$; hence, $[1 / 1-b]\left([a+c] d_{\theta}\left(x_{n+1}, x_{n}\right)-[1-b] d_{\theta}\right.$ $\left.\left(x_{n+2}, x_{n+1}\right)\right) \in C$, that is, $[a+c] /[1-b] d_{\theta}\left(x_{n+1}, x_{n}\right)$ $d_{\theta}\left(x_{n+2}, x_{n+1}\right) \in C$. Therefore, we have

$$
d_{\theta}\left(x_{n+2}, x_{n+1}\right) \prec \frac{[a+c]}{[1-b]} d_{\theta}\left(x_{n+1}, x_{n}\right), \quad n=0,1,2, \ldots
$$

Since $[a+c] /[1-b]<1, \quad d_{\theta}\left(x_{n+1}, x_{n}\right) \longrightarrow \stackrel{d_{\theta}}{\longrightarrow} \Theta$, and using Lemma 7 proves that the sequence of iterates is a Cauchy sequence. According to the completeness of $X$, there exists $x \in X$ such that $x_{n} \longrightarrow{ }_{n \rightarrow \infty}^{d_{\theta}} x$ and $\lim _{n \longrightarrow \infty} d_{\theta}$ $\left(x_{n}, x\right)=\Theta$; we show that $x$ is a fixed point of $S$. In fact, we have

$$
\begin{aligned}
& d_{\theta}\left(x_{n+1}, S(x)\right)=d_{\theta}\left(S\left(x_{n}\right), S(x)\right), \quad n=0,1,2, \ldots \\
& \prec a d_{\theta}\left(x_{n}, x\right)+b d_{\theta}(x, S(x))+c d_{\theta}\left(x_{n}, S\left(x_{n}\right)\right) \\
& \prec a d_{\theta}\left(x_{n}, x\right)+b d_{\theta}(x, S(x))+c d_{\theta}\left(x_{n}, x_{n+1}\right) \\
& \longrightarrow{ }_{n} \operatorname{bd}_{\theta}(x, S(x)) \text {, }
\end{aligned}
$$

$$
\begin{aligned}
d_{\theta}(x, S(x)) \prec & \theta\left(d_{\theta}\left(x, x_{n}\right), d_{\theta}\left(x_{n}, S(x)\right)\right) \\
\prec & \theta\left(d_{\theta}\left(x, x_{n}\right), \theta\left(d_{\theta}\left(x_{n}, x_{n+1}\right), d_{\theta}\left(x_{n+1}, S(x)\right)\right)\right) \\
& \longrightarrow \longrightarrow \rightarrow \infty\left(\Theta, \theta\left(\Theta, \lim _{n \longrightarrow \infty} d_{\theta}\left(x_{n+1}, S(x)\right)\right)\right) \\
& \prec \theta\left(\Theta, \lim _{n \longrightarrow \infty} d_{\theta}\left(x_{n+1}, S(x)\right)\right) . \\
& \prec \lim _{n \longrightarrow \infty} d_{\theta}\left(x_{n+1}, S(x)\right) .
\end{aligned}
$$

Using inequalities (41) and (42) proves that $d_{\theta}(x, S(x)) \prec b d_{\theta}(x, S(x))$; hence, $(b-1) d_{\theta}(x, S(x)) \in C$, and consequently, $d_{\theta}(x, S(x))=\Theta$ because $C \cap-C=\{\Theta\}$, that is, $S(x)=x$. Finally; we prove that $x$ is the unique fixed point of $S$. Suppose, on the contrary, that $x$ and $y$ are two distinct fixed points of $S$. So, we get the following: 


$$
\begin{aligned}
d_{\theta}(x, y)= & d_{\theta}(S(x), S(y)) \prec a d_{\theta}(x, y)+b d_{\theta}(x, S(x)) \\
& +c d_{\theta}(y, S(y))=a d_{\theta}(x, y) .
\end{aligned}
$$

Hence, $\left[a d_{\theta}(x, y)-d_{\theta}(x, y)\right] \in C$ and $[a-1] d_{\theta}$ $(x, y) \in C$, but $[a-1]<0$; this implies that $d_{\theta}(x, y)=\Theta$ because $C \cap-C=\{\Theta\}$, that is, $x=y$.

\section{Conclusion}

In this paper, we generalize the concept of $\theta$-metric space to the concept of the $\theta$-cone-metric space as a generalization of a metric by replacing the triangle inequality with a more generalized inequality using some $\theta$-action functions and replacing the usual ordered relation of real numbers by ordered relation induced by a cone in a normed space. We investigate the convergence and Cauchyness in such a $\theta$-cone-metric space. Furthermore, we prove some lemmas and use them to give further generalizations of some wellknown fixed point theorems in this context [14].

\section{Data Availability}

No data were used to support this study.

\section{Conflicts of Interest}

The author declares no conflicts of interest.

\section{Authors' Contributions}

The sole author contributed $100 \%$ to the article. The author read and approved the final manuscript.

\section{References}

[1] L.-G. Huang and X. Zhang, "Cone metric spaces and fixed point theorems of contractive mappings," Journal of Mathematical Analysis and Applications, vol. 332, no. 2, pp. 1468-1476, 2007.

[2] H. Liu and S. Xu, "Cone metric spaces with banach algebras and fixed point theorems of generalized lipschitz mappings," Fixed Point Theory and Applications, vol. 2013, Article ID 320, 2013.

[3] R. H. Haghi, S. h. Rezapour, and N. Shahzad, "Some fixed point generalizations are not real generalizations," Nonlinear Analysis, vol. 74, pp. 1799-1803, 2010.

[4] H. Cakally, A. Sonmez, and C. Genc, "On an equivalence of topological vector space valued cone metric spaces and metric spaces," Applied Mathematics Letters, vol. 25, pp. 429-433, 2012.

[5] W.-S. Du, "A note on cone metric fixed point theory and its equivalence," Nonlinear Analysis: Theory, Methods \& Applications, vol. 72, no. 5, pp. 2259-2261, 2010.

[6] M. Abbas, V. Cojbašić Rajić, T. Nazir, and S. Radenović, "Common fixed point of mappings satisfying rational inequalities in ordered complex valued generalized metric spaces," Afrika Matematika, vol. 26, no. 1-2, p. 17, 2013.

[7] F. Khojasteh, E. Karapinar, and S. Radenovic, " $\theta$-metric space: a generalization," Mathematical Problems in Engineering, vol. 2013, Article ID 504609, 7 pages, 2013.
[8] T. Suzuki, "The strongest sequentially compatible topology on a $\nu$-generalized metric space," Journal of Nonlinear Variable Analysis, vol. 1, pp. 333-343, 2017.

[9] S. M. A. Abou Bakr, "Common fixed point of generalized cyclic banach algebra contractions and banach algebra kannan types of mappings on cone quasi metric spaces," J. Nonlinear Sci. Appl.vol. 12, no. 10, pp. 644-655, 2019.

[10] S. M. A. Abou Bakr, "A study on common fixed point of joint $(A ; B)$ generalized cyclic $\phi-a b c$ weak nonexpansive mappings and generalized cyclic coabc; $r$ ntractions in quasi metric spaces," Abstract and Applied Analysis, vol. 2020, pp. 7-13, Article ID 9427267, 2020.

[11] S. Banach, "Sur les opérations dans les ensembles abstraits et leur application aux équations intégrales," Fundamenta Mathematicae, vol. 3, pp. 133-181, 1922.

[12] S. Reich, "Kannan's fixed point theorem," Bollettino dell'Unione Matematica Italiana, vol. 4, pp. 1-11, 1971.

[13] G. E. Hardy and T. D. Rogers, "A generalization of a fixed point theorem of reich," Canadian Mathematical Bulletin, vol. 16, no. 2, pp. 201-206, 1973.

[14] J. Fernandez, N. Malviya, S. Radenovi'c, and K. Saxena, "F-cone metric spaces over Banach algebra," Fixed Point Theory and Applications, vol. 7, 2017. 